\title{
Affective E Learning Model for Recognising Learner Emotions
}

\author{
T. C. Sandanayake, A. P. Madurapperuma, and D. Dias
}

\begin{abstract}
Online learning is commonly accepted as a support tool for educators as well as a medium of delivery of any-time, any-where content of a wide range of study programs to a widely dispersed learner community. Web-based learning environments are a relatively new medium of learning to Sri Lankan universities. Characteristics of learners of such environments vary widely, from technology geniuses to technological novices, from high bandwidth access to slow Internet connections etc. There are predictions of a near future boom of digital learning in Asian educational context, challenging the conventional face to face learning environments. Like any learning process, digital learning depends on effective communication of human knowledge, whether this occurs in a face-to-face classroom or across the Internet. The effectiveness of digital learning also depends on establishing two-way communication between facilitators and learners, and among learners themselves. Within the state university settings, learners experience varied emotions and interest towards learning. Although both emotions and interest can increase learners' likelihood to engage in traditional learning, little is known about the influence of emotions and interest in learning activities in a digital environment. Previous research have identified that emotions occur while individuals assess events in their environment that are related to the needs, goals and well-being. Moreover, recent research on the emotional response to online learning has focused on the importance of learners' feelings in relation to the community of learning. Major objective of this research study is to introduce a new model of online learning with relevance to the emotional response of the learning community. The study is based on Barry Kort's Learning Spiral Model which is a four quadrant learning model in which emotions change while the learner moves through quadrants and up the spiral
\end{abstract}

Index Terms-Emotions, learning environment, online learning, online learner

\section{INTRODUCTION}

Emotion is one of the most controversial topics in psychology and it plays a vital role in many human activities. Research in neurosciences and human psychology has identified and proved that emotions exert influences in various behavioral and cognitive processes of a human being,

Manuscript received October 15, 2011; revised October 30, 2011.

T. C. Sandanayake is a lecturer in the Faculty of Information Technology of University of Moratuwa, Sri Lanka and currently reading Master of Philosophy Degree at the University of Morauwa (Phone : +94717646462 , email : thanujas@uom.lk).

A. P. Madurapperuma holds a Ph.D in computer science and currently serving as the Deputy Director (Admin) at the Keio-NUS CUTE Center at National University of Singapore (Phone : +6590235340, email. ajitolanka@gmail.com).

D. Dias is a professor at the Department of Electronics and Telecommunications of University of Moratuwa, Sri Lanka (Phone: +94-11-2650634, email : Dileeka.Dias@uom.1k). such as decision making, motivating, long-term memorizing [1]. Human learning may occur as part of education, personal development and learning should always be goal-oriented and motivated. Education in the largest sense is any act or experience that has a formative effect on the mind, character or physical ability of an individual ([2], [3]). The linkage between complex learning and emotions has received increasing attention in the fields of psychology, education, neuroscience and information technology ([4], [5], [6], [7]) A deep understanding of such affective learning connections is needed in order to design engaging educational artifacts that range from responsive intelligent tutoring systems [7].

Goleman [8] has mentioned in the book, Emotional Intelligence, that expert teachers are able to recognize a student's emotional state and respond in an appropriate manner that has a positive impact on the learning process. In the past decade, e-learning has evolved from Computer Aided Instruction, through Intelligent Tutoring System, to Smart Classrooms, and to Mobile Learning anytime ([9], [10]). During the last decade researchers in education have made extensive progress towards identifying the cognitive processes that are significant to learning ([11], [12]). Continuous and increasing exploration of the complex set of parameters surrounding online learning reveals the importance of the emotional states of learners and especially the relationship between emotions and effective learning [10]. The significance of the emotional aspects in higher education has also been recognized and systematic inquiry of emotions in the learning process has been slow to emerge so far [13].

\section{Theoretical Framework AND PREVIOUS StUdies}

E-learning has become a learner-centered, personalized learning technology. Only a few explorations into the extent to which emotions are associated with learning on line are reported ([11], [13]). Most of the past research has been on the physiological factor of the learner, based on the traditional class room face to face learning ([9], [11]). E-learning has been depicted as less emotional and more impersonal or as lacking in emotional richness (e.g., lack of body language, facial expressions, and gestures) when compared to face-to-face learning [14]. Adult learners experience on affective learning is in a range from positive and energizing to negative [13]. One of the most ubiquitous notions in human psychology is that some emotions have a special status when categorized as basic, primary, or fundamental emotions [4]. The approaches behind recognizing learner behavior has evolved since 1970s and two approaches that compete most predominantly in the area of education have been identified ([8], [15]). The first one is 
the basic categorical structure and the second is dimensional structure [8].

The basic categorical approach was one of the earliest to be developed and was applied by Plutchik [6]. According to Plutchik [6], the eight primary emotions are joy, acceptance, surprise, fear, sorrow, disgust, anticipation and anger [16]. Plutchik arranges them in a circular pattern, which he likened to a colour wheel.

The second approach to defining emotional structure is the dimensional approach, which says all emotions can be identified as displaying association with one or more dimensions, such as intensity, pleasantness or degree of activation [15]. Most often, the two broad dimensions of pleasure and arousal have been identified using factor analysis and multidimensional scaling (MDS) techniques. In this model emotions are not clustered along the axes but are arranged in a circular pattern around the dimensions, hence being referred to as a circumplex. This dimensional approach has been identified by James Russell in 1979- 1980. Russsel invented the circumplex model of representing emotions. Affective e-learning model is explored by Liping Shen, Minjuan [10] Wang and Ruimin Shen in Shanghai China. This study has investigated how emotions evolve during the learning process and how emotion feedback could be used to improve learning experience using emotion detection technologies from biophysical signals. The research study was guided by Russell's Circumplex Model of Affect [17] and Kort's Learning Spiral Model ([18], [19]).

\section{THE RESEARCH PROBLEM}

Modern education system uses multiple methods and tools such as electronic learning, mobile learning, problem based learning, project based activities, online discussion groups and chat forums to facilitate learning. Today, many reputed higher education institutions allow their learners to acquire knowledge and skills in open and distance mode giving the learners flexibility in time, location and their own pace[20]. Adult learners especially prefer to have more freedom in the learning phase [21]. One of the major problems in distance learning is the lack of methods to recognise learner behavior. Therefore, the current study addresses the problem of recognizing e learner's emotional state during learning. The same problem has been addressed in much research on face to face environment but not on distance learning environment. This research model will help to identify e learner's emotional state with respect to their level of learning.

\section{AIM OF THE RESEARCH STUDY}

The aim of the research study is to build a model to recognize the e learner's emotional state with respect to their learning performances. The proposed e learning model will address the issue of not recognizing and responding to e learner's emotions while they learn. This model will also be helpful to identify the e learners' learning performances while measuring the emotional state.

\section{Proposed Affective LeARning Model}

The research study has been designed aiming the first and second year undergraduates following a B.Sc. (honours) degree in Information Technology. The degree programme is offered in a face to face environment and with some course work done in an online mode. The lessons used for the research study are conducted in both open and distance environments and Moodle has been used as the Learning Management System (LMS). The observations collected through the LMS are used to evaluate the learner's level of learning. Research experiment are carried out in different online activities such as, reading assessment, quiz, online discussion forums, chat forums, lesson assignments etc. The learning time and other time related calculations are measured through the Moodle LMS and grades and marks for the evaluation activities are calculated after the teacher evaluation.

\section{A. System Architecture of the Research Study}

Online learning comprises many forms of technology supported learning and teaching providing socio and technological association. The learner facilitation gets improved through online learning; perhaps improved since the concepts of affective computing embedded into it. The combination of technology enhanced learning and affective computing can be applied to the online learning community. The current context of online learning has no provision to recognize learner's emotions while they learn unless it observe the bio-physical signals of a learner ([3], [12]). The biological information of a learner should be observed through highly confirmed and controlled learning environment and the provision to practice this scenario in open and distance learning environment is quite difficult. Therefore, some other architecture should be followed in measuring such emotions. The current research study proposes a solution to recognize learner emotions in real time environment without measuring any bio-physical signals. The high level system architecture of the proposed solution is shown in Figure 1.

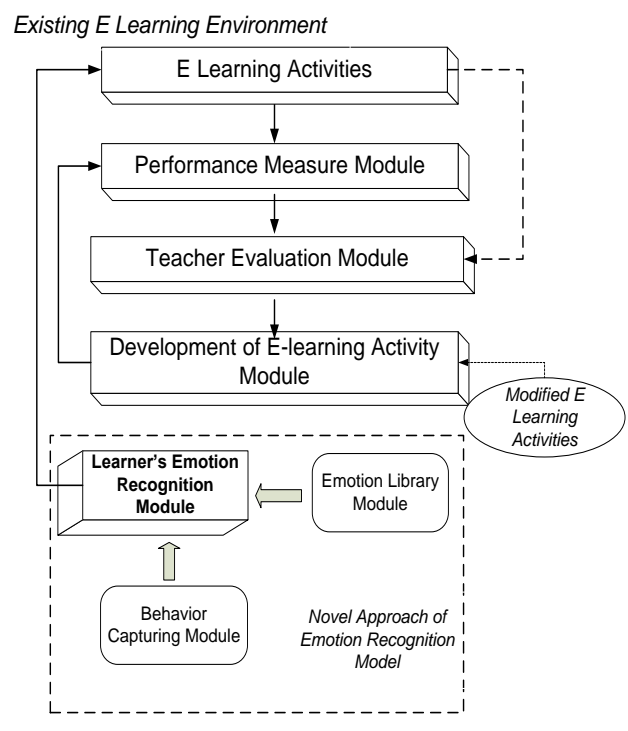

Fig. 1. High level system architecture 
The system consists of different modules in online learning. The upper part of the diagram discusses how the existing online learning environment has been established and the lower part discusses the novel approach to the online environment. The existing e learning module consists of three major modules: performance measure module, teacher evaluation module and development of e learning activity module. The novel approach which consists of the learner emotion into consideration is being included in the lower part which comprises two major modules naming emotion library module and behaviour capturing module.

\section{B. Diversified Online Learning Activities - Existing \\ Online Learning Environment}

This section describes how the existing learning activities takes place in most of the online learning programmes. In Figure 1, the e learning activity module consists of different activities such as reading, online lectures, quizzes, discussion forums, chat forums and example based learning. The learning methods should be constructive to make online learning courses effective. Such methods include constructive discussions on knowledge enhancement, interactive simulation, problem solving scenarios, project based method, collaborative exercises etc [20]. Emotions are experienced during computer-mediated interactions such as those occurring in the online environment. In the online environment it is acknowledged that the learner behavior or associated affective reactions are not monitored in the same way as in conventional systems. When online learning is used for collaborative purposes, the affective reactions experienced while working in the environment result mainly from the interactions between the learners [20]. One of the most important activities among online teaching and learning is performance evaluation or assessment. As given in the Figure 1, the second module discusses the performance evaluation of the online learning structure. Researchers argue that the learner evaluation or assessment in online learning is essentially no different from any other form of learning [20, 21].

Practicing effective assessment activities in online learning is a challenging task for any academic. Appropriate assessment techniques are essential to assess the learner's knowledge level and also to evaluate the teaching methodologies. Irrespective of all the performance evaluation methods, there is no such mechanism to evaluate the learner's emotional feedback together with the graded assessments ([1], [11]). Teacher evaluation module discusses how teachers themselves can evaluate the teaching methodologies. Most of the time in conventional online learning the learners' examination results, online course feedback systems and the learner requests are used as the teacher evaluation methods. The student's emotional feedback is not used as a tool for measuring teacher performances in online teaching. Therefore the problem of addressing the online learner emotions will be addressing once the proposed systems. The technologies and approaches that constitute online learning are diverse, often blended with conventional approaches. So it is difficult to provide a single approach to evaluating impact.

\section{Learner Emotion Recognition Model}

The fundamental concept of learner behavior and their emotions have been addressed by different philosophers and scientists in the past. Many of the past research activities were carried out with the basis of capturing the learner's emotions using bio physical signals. This research study is based on recognizing learner emotions during the e learning process without accessing any of the biological signals as an input. Therefore, the novel approach can be used in both open and distance environment where the learner is physically invisible to the system. As per given in the Figure 1, the emotion recognition module consists of two sub module called emotion library module and emotion recognition thorough behavior capturing module.

\section{Emotion Library Module}

Figure 2 shows the emotion library module. It incorporates methodologies to use affective computing and kansei engineering to identify emotions. At the same time theories described in the education and as well as human psychology also added to this module. The data pertaining to current studies on online learning are used as a powerful resource in this research. Current study aims to explore the learner behavior using Barry Kort's Learning Spiral Model by observing and analyzing the learning models described in numerous areas as given in the library. The Kort's learning spiral module is depicted in Figure 3.

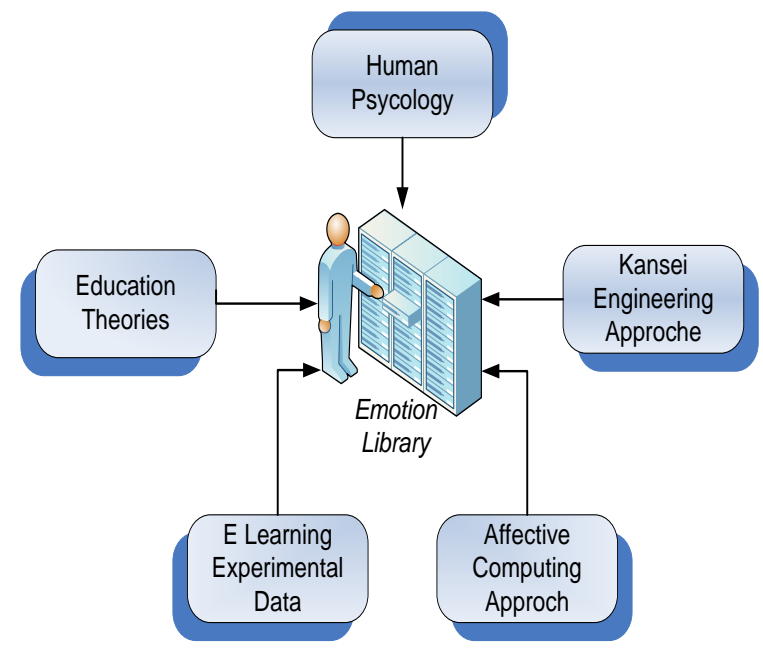

Fig. 2. Emotion library module

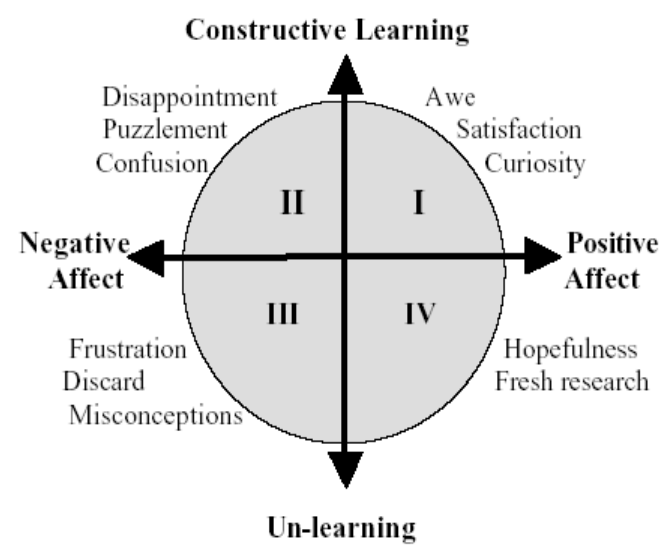

Fig. 3. Kort's learning spiral model (kort, 2001) 


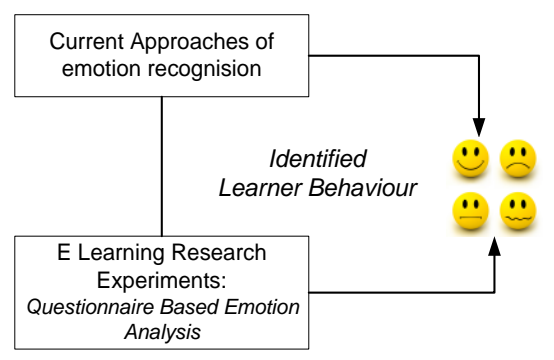

Fig. 4. Emotion recognition through learner behavior

Barry Kort has used emotion detection technologies from biophysical signals, and explored how emotion evolves during the learning process and how emotion feedback can be used to improve learning experiences. Kort ([18], [19]) proposed a four quadrant learning spiral model in which emotions change while the learner moves through quadrants and up the spiral. Figure 3 shows Kort's Spiral model of affective learning. This model has covered the spectrum of areas which had to be developed in the education methodologies. The Figure 3 shows the relationship between learners' emotions and level of learning. The horizontal axis of the Figure 3 denotes emotion axis and the vertical axis denotes learning axis. The positive (more pleasurable) emotions are on the right side where the negative (more unpleasant) emotions are on the left side. The vertical axis symbolizes the construction of knowledge upward and the discarding of misconceptions downward ([18], [19]). The learners are experiencing positive affect and constructing knowledge in quadrant I. The learners move to quadrant II once the discrepancies appear between learner's knowledge structure and the information provided. This part consists of constructive learning and negative affect and then the learners experience affective states such as confusion. When a learner tries to sort out a puzzle but fails, the learner might move into quadrant III. This is the quadrant of unlearning and negative affect, when the learner is experiencing states such as frustration [10]. After the misconceptions are discarded, the learner moves into quadrant IV, marked by unlearning and positive affects $[3,10]$.

\section{E. Recognising Learner Emotions - Achievement Emotion Questionnaire for Online Learning}

The second sub module of the learner emotion recognition module is the learner emotion identifying module shown in Figure 4. The basic question of "what are emotions?" has been addressed by several psychologist, anthropologists and sociologist since centuries. The concept of academic emotions are evolved since three decades and it has been described as the emotional experiences associated with learning ([17], [18], [19]). In the educational context emotions are playing a major role in learner performances and both positive and negative emotions affected learners attention, motivation, limits and performances in an optimal way ([6], [14], [16]). This research study is mainly aiming to study the emotions of online learners in the context of higher education. Here the study aims to describe how the learner behavior can be recognized with respect to the Kort's learning spiral model. Here the module excludes the use of biological signals to recognize emotions due to difficulties in collecting such data in practice. The model has build based on the observations of facial expressions, gross body languages and the content of the tone of speech. Testing emotions in an online learning environment using biological signals has to be tested in a highly controlled environment. Therefore this research study has discarded the methodology of using biological signals as inputs. The alternate methodology of detecting emotions then has to be a self reported instrument which can be applicable in real time online environment.

The Achievement Emotion Questionnaire in a multidimensional self reported instrument which has designed to assess the achievement emotions of the learners in higher educational context [22]. Even though this method has been designed to examine mostly cognitive dimensions of the learning process, they have also been used to study emotions with respect to the online learning ([18], [19]). Both qualitative and quantitative approach has been used in the analysis of data to generate the results. The AEQ has developed in the context of higher education based on class room teaching environment and in this research study this has been modified to online learning environment. Therefore the tool often can call as AEQ for online learning which measures a number of discrete emotions for each three mail academic situations that is engaging in LMS, learning online and facing test and exams.

The tool consists of set of questions to measure four positive emotions (enjoyment, pride, hope and relief) and five negative emotions (anger, anxiety, hopelessness, shame and boredom). The rationale behind testing such emotions in the tool is that these are the emotions which are frequently asses by the college students. The second main reason is that the selected emotions can be group according to two dimensions of valance (positive vs. negative) and activation (activating vs. deactivating) [22]. The questions of the questionnaire tool has categories based on six different sections in online learning such as on line access, online familiarisation, reading activities, learning through online community, online assessments, lesson break activities.

The AEQ for online learning represents emotions for each of four categories as positive activating: enjoyment, hope pride; positive deactivating: relief; negative activating: anger, anxiety, shame; negative deactivating: hopelessness, boredom [22]. The study is mainly aiming to assess online learners' individual emotional reactions in the achievement situations which have been termed as trait achievement emotions in learning. Within the each section of online learning the emotions are being tested in three different situations and that is before, during and after learning. The AEQ for online learning scored in five point Likert scale starting form "strongly disagree" to "strongly agree". In analysing the results of the survey has been used the confirmatory factor analysis for the original version of AEQ [22]. The instruction manual of the AEQ for online learning request the online learners to describe their general typical emotional experiences occur during the learning process and engaging the activities of online learning.

\section{F. Measuring the Level of Learning}

As described in the Barry Kort's learning spiral model, affect recognizing and finding the exact quadrant has to be 
done together by measuring the learning level of the learners. The current research study uses different parameters to measure the learning level of the online learner and it will be evaluated at the end of the learning process. The parameters have been selected based on the past research carried out on online learning and the performance measurement of the learners. Therefore the observable measurements without disturbing the learner physically have been identified as the parameters of the level of learning. The six parameters are as follows;

$X_{1}$ : Average Deviation from expected time spent on each learning activity

$X_{2}$ : Average time spent on lesson break activities

$X_{3}$ : Average Quiz marks

$X_{4}$ : Average marks for the discussion forum

$X_{5}$ : Average time spent on chat forum

$X_{6}$ : Average time spent on browsing and navigation in the LMS

Multiple regression analysis will be used to analyse the leaner's level of learning in the learning environment. The purpose of using multiple regression analysis in the research study is that to recognize the relationship between several independent variables as given above $X_{1}$ to $X_{6}$. More precisely, multiple regression analysis helps us to predict the value of $\mathrm{Y}$ for given values of $X_{1}, X_{2}, \ldots, X_{6}$. Hence, we use the following regression model for the analysis.

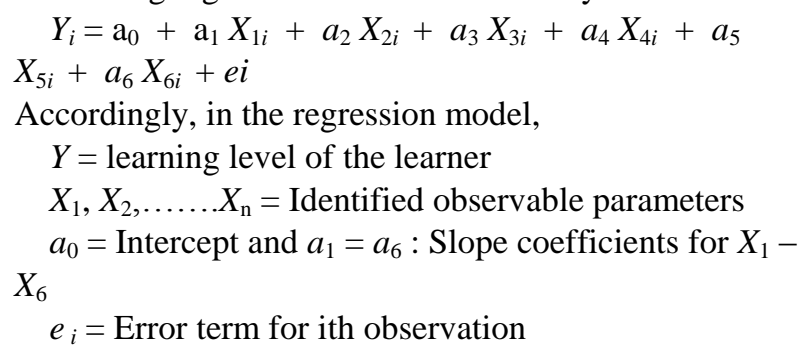

To apply the equation, each $X_{j}$ score for an individual case is multiplied by the corresponding aj value, the products are added together. The constant $\mathrm{a} 0$ and ei the error term is added to the sum. The result is $Y_{i}$, the predicted $Y i$ value for the case The constructive learning of the lesson will be measure by conducting online assessments which will be marked and graded by the teacher. The learning material has to be evaluated by a pedagogy expert. The marks obtained by the learner for the given assessment will be considered as proportional to the learner's level of learning. Therefore, that mark can be used as a measure of level of the learning. Finally, once the regression model gets completed with the numeric values for a1 to a6 the learners learning level can be measured by feeding the values to the observable parameters $X_{1}$ to $X_{6}$.

\section{CONCLUSIONS}

Emotions are important in adult learning because they can either impede or motivate learning. Electronic learning has been portrayed as less emotional and more impersonal or as lacking in emotional richness when compared to face-to-face learning since the learner is physically invisible to the teacher. Online education programs have enabled learning opportunities to learners that allow for breaking away from space and time constraints. In Sri Lankan context this problem becomes worse since the online delivery mode is quite new for the higher educational system. The aim of the research study is to build a model to recognize the e learner's emotional state with respect to their learning performances. The proposed e learning model addresses the issue of not recognizing and responding to e learner's emotions while they learn. This model will also be helpful to identify the e learners' learning performances while measuring the emotional state. The research study has been designed aiming the undergraduate learners following an honours degree in Bachelor of Science in IT, University of Moratuwa The high level architecture of the proposed system describes the connectivity of the existing online environment and the novel approach of online learning which enables affect recognition. Current study explores the learner behavior using Barry Kort's Learning Spiral Model. This article proffers a novel model by which to conceptualize the impact of emotions upon learning with the application of Barry Kort's learning spiral model. This model is described by two major constructs which are the learning level of individual learner and the emotional state of the learner. The research study measures both the elements individually and separately. When measuring the learning level of the learner, the study will be using six observable parameters of online learner. Multiple regression analysis will be used in measuring the level of learners. The emotional element of the leaner will be measured using multidimensional self reported instrument which assesses online learner's individual emotional reactions in achievement situations. The tool is termed as Achievement Emotion Questionnaire for Online Learning and four positive emotions and five negative emotions are measured in the real time online learning environment. The AEQ for online learning is scored in five point Likert scale and items included in the questionnaire were based on learner's emotional experiences experimented by Rainhard Pekrun. The questions are categorised in to six different sections in online learning and assesses the emotional state of the learner. Finally the learner's emotional state and the level of learning is mapped in two dimensional space and the responses are used to enhance leaner performances.

\section{REFERENCES}

[1] S. Chaffar, C. Frasson, "Inducing Optimal Emotional state for learning in Intelligent Tutoring Systems" In proceeding of International Conference of Intelligent Tutoring System, Maceio, Brazil, 2004.

[2] Wikipedia, Education, http://en.wikipedia.org/wiki/Education, 2010

[3] R. W. Picard, “Affective computing”, Boston: MIT Press, 1997

[4] P. Ekman, Are there basic emotions?, 99(3): 550-553, 1992.

[5] G. Gibbs, "Improving the quality of student learning". Oxford, UK: Oxford Centre for Staff Development, 1992

[6] R. Plutchik, "Emotions: Psychoevolutionary Review", Harper and Row, ISBN 0-06-045235-8, 1980

[7] A. Graesser, S. K. D Mello., S. D Craig, J. S. Witherspoon, J. Sullins, B. Mc Danial, B. Gholson, "The Relationship between Affective States and Dialog Patterns during Interactions with AutoTutor", 2007

[8] D. Goleman, "Emotional Intelligance", NY, 1995

[9] T. C Sandanayake, A. P. Madurapperuma, "Conceptual Model for E-Learning Systems Using Kansei Engineering Techniques, A Preliminary Study", International Multi Conference on Biometrics and Kansei engineering" (ICBAKE- KEAS), Poland, pp. 148 - 152, 2009

[10] L. Shen, M. Wang, and R. Shen, Affective e-Learning: "Using "Emotional" Data to Improve Learning in Pervasive Learning Environment." Journal of Educational Technology and Society, 12 (2), pp. 176-189,2009 
[11] T. C. Sandanayake, A. P. Madurapperuma, "Emotional Reactions of Undergraduates in Digital Learning Environment", International Conference on Information Technology, e Asia, Sri Lanka, 2009

[12] J. M. Dirkx, "Engaging emotions in adult learning: A Jungian perspective on emotion and transformative learning", New Directions for Adult and Continuing Education, 109,15-26, 2006

[13] T. C. Sandanayake, A.P. Madurapperuma, "Emotional Reactions of Undergraduates in Digital Learning Environment",e Asia 2009 (International Conference on Information Technology), Sri Lanka, 2009

[14] M. Zembylas, "Adult learners' emotions in online learning", International Journal of Distance Education, Vol. 29, pp. 71-87, 2008

[15] P. Darbyshire, R. Bell, H. McDonald, "Testing the Circumplex Model of Emotions in a Consumer Setting", http:// /www/anzmac2006/documents/Darbyshire_Penny, 2006

[16] R. Plutchik, "A general psychoevolutionary theory of emotion,' in Emotion Theory, Research, and Experience" (R. Plutchik and H. Kellerman, eds.), vol. 1, Theories of Emotion, Academic Press., 1980

[17] J. A. Russell, "A circumplex model of affect", Journal of Personality and Social Psychology: 39, 1161-1178, 1980

[18] B. Kort, R. Reilly, and R. W. Picard, "An affective model of interplay between emotions and learning: Reengineering educational pedagogy-building a learning companion", Proceedings of the IEEE InternationalConference on Advanced Learning Technologies, Los Alamitos: CA: IEEE, 43-46. 2001

[19] B. Kort, R. Reley, "The science behind the art of teaching. Science: emotional state and learning". Massachusetts Institute of Technology, SITE, pp. 3021 - 3026, 2004

[20] G. S. Lunney, "Emotional Intelligence", http://www.selfgrowth.com/articles/Lunney1.html, 2007

[21] T. C. Sandanayake, A. P. Madurapperuma., "Enhancing the Learning Environment by Identifying the Learner Behavior in an e-Learning System"., Int. Conference on Engineering Education and Research, Korea, 2009

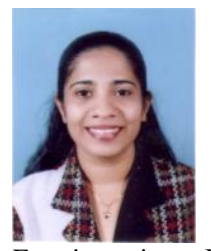

Thanuja Chandani Sandanayake is a Lecturer in the Faculty of Information Technology of University of Moratuwa, Sri Lanka since 2005. She has obtained B.Sc in Physical Sciences from the University of Wayamba, Sri Lakna in 2004 and currently reading Masters of Philosophy degree under the topic of "Developing a Computational Model for Recognizing Students'

Emotions in e-Learning Systems" at University of Moratuwa. Ms Sandanayake is having over four years of experience in research and teaching and possesses sound knowledge in e Learning, Management, Information Technology, Research Methodologies, Kansei Engineering and Affective Systems.

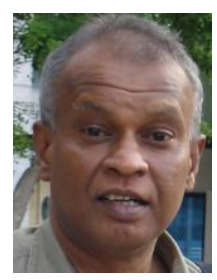

Ajith Parakum Madurapperuma is the Deputy Director (Admin) at Keio-NUS CUTE Center at National University of Singapore since 2009. Before joing NUS he has served as a senior lecturer in the Faculty of Information Technology, Univerisuty of Moratuwa and visiting lecture of Department of Computer Sciences and Engineering, University of Moratuwa and Open University of Sri Lanka.

Dr. Madurapperuma has obtained ISPJAE, Havana, Cuba in 1987, M.Sc. in Computer Science and Ph.D from University of Wales, Cardiff. He has served as the dean of the Faculty of Information technology from 2003-2006.

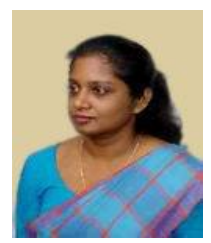

Dileeka Dias is a professor at the Department of Electronics and Telecommunication of University of Moratuwa, Sri Lanka. She has obtained B.Sc.in Engineering from University of Moratuwa, M.Sc and Ph.D qualifications from California. Prof. Dias is a charted engineer and member of IEEE who served as the dean of the faculty of Information Technology of university of Moratuwa from 2006-2009. Main research interests of Prof. Dileeka Dias are Broadband and Wireless Communications, DSP-based System Design 